1. Pulsinelli WA, Waldman S, Rawlinson D, Plum F. Moderate hyperglycemias auments ischemic brain damage: A neuropathologic study in the rat. Neurology 1982; 32: 1239-46.

2. Combs DJ, Reuland DS, Martin DS, Zelenock GB, D'Alacy LG. Glycolytic inhibition by 2-deoxyglucose reduce hyperglycemic associated mortality and morbidity in the ischemic rat. Stroke 1986; 17: 989-94.

3. De Courten-Myers GM, Yamaguchi OS, Myer RE. Accentuation of hypoxic/anoxia brain injury by postexposure infusion of glucose solutions. Neurology 1982; 31: A110.

4. De Courten-Myers. Hyperglycemia enlarges infarct size in cerebrovascular occlusion in cats. Stroke, 1988; 19: 623-30.

5. Vázquez-Cruz J, Martí-Vilalta JL, Ferrer I, Pérez-Gallofré A, Folch J. Progressing cerebral infarct in relation to plasma glucose in gerbils. Stroke 1990; 21: 1621-4.

6. Dietrich WD, Alonso O, Busto R. Moderate hyperglycemia worsens acute blood-brain barrier injury after forebrain ischemia in rats. Stroke 1993; 24: 111-6.

7. Araki N, Greenberg J, Sladky J, Uematsu D, Karp A, Reivich M. The effect of hyperglycemia on intracellular calcio in stroke. J Cereb Blood Flow Metab 1992; 12: 469-76.

8. Paljärvi L, Renchrona S, Soderfeldt B, Olsson Y, Kalimo H. Brain lactic acidosis and ischemic cell damage: Quantitative ultrastructural changes in capillaries of rat cerebral cortex. Acta Neuropathol (Berl) 1983; 60: 232-40.

9. Duverger D, MacKenzie ET. The quantification of cerebral infarction following focal ischemia in the rat: Influence of strain, arterial pressure, blood glucose, concentration and age. J Cereb Blood Flow Metab 1988; 8: 449-61.

10. Pulsinelli WA, Levy DE, Sigsbee B, Scherer PG, Plum F. Increased damage after ischemia stroke in patients with hyperglycemia with or without established diabetes mellitus. Am J Med 1983; 74: 540-4.

11. Peinado E, Ozaita G, Antigüedad A, Calandre L, Bermejo F. La glucemia como factor pronóstico en el infarto cerebral. Neurología 1988; 3: 50-3.

12. Castillo J, Martínez F, Leira R, Prieto JM, Lema M, Noya M. Mortality and morbidity of acute cerebral infarction related to temperature and basal analytic parameters. Cerebrovasc Dis 1994; 4: 66-71.

13. Giroud H, Dumas R. Hyperglicemie a la phase initiale d'un accident vasculaire cerebral. Indice de mauvais pronostic. Presse Med 1987; 16: $1079-82$

14. Candelise L, Landi G, Orazio EN, Boccardi E. Prognostic significance of hyperglycemia in acute stroke. Arch Neurol 1985; 42: 661-3.
15. Cazzato G, Zorzon M, Mase G, Iona LG. Hyperglycemia at ischemic stroke onset as prognostic factor. Ital J Neurol Sci 1991; 12: 283-8.

16. Toni D, Sachetti ML, Argentino C, Gentile M, Cavalleti C. Does hyperglycemia play a role on the outcome of acute ischemic stroke patients? J Neurol 1992; 239: 382-6.

17. Abu-Zeid HAH, Choi NW, Hsu PH, Maini KK. Prognostic factors in the survival of 1484 stroke cases observed for 30 to 48 months. II. Clinical variables and laboratory measurements. Arch Neurol 1978; 35: 213-8.

18. Gray C, Shaw D, Cartlidge NE; Bates D. The prognostic value of stress hyperglicemia and previously unrecognizable diabetes in acute stroke. Diabet Med 1987; 4: 237-40.

19. Stoute F. Ischemic stroke made worse with intravenous glucose. Ann Emerg Med 1986; 15: 1121.

20. Ginsberg MD, Welsh FA, Budd WW. Deleterious effect of glucose pretreatment on recovery from diffuse cerebral ischemia in the cat. Stroke 1980; 12: 347-54.

21. Castillo J, Dávalos A, Naveiro J, Noya M. Neuroexcitatory amino acids and their relationship to infarct size and neurological deficit in ischemic stroke. Stroke 1996; 27: 1060-5.

22. Wagner KR, Kleinholz M, De Courten Myers GM. Myers RE. Hyperglycemic versus normoglycemic stroke: Topography of brain metabolites, intracellular $\mathrm{pH}$, and infarct size. J Cereb Blood Flow Metab 1992; 12: 213-22.

23. Pulsinelli WA. Experimental hyperglycemia and diabetes mellitus worsen stroke outcome. Trans Am Neurol Assoc 1980; 105: 21-4.

24. Berger L, Hakim A. The association of hyperglycemia with cerebral edema in stroke. Stroke 1986; 17: 865-71.

25. Duchrow RB, Beard DC, Brennan RW. Regional cerebral blood flow decreases during hyperglycemia. Ann Neurol 1985; 17: 267-72.

26. Welsh FA, O'Connor MJ, Marcy VR, Spatacco AJ, Johns RL. Factors limiting regeneration of ATP following temporary ischemia in cat brain. Stroke 1982; 13: 234-42.

27. Woo J, Ma JT, Robinson JD, Phil M, Yu YL. Hyperglycemia is a stress response in acute stroke. Stroke 1988; 19: 1359-64.

28. Van Kooten F, Hoogerbrugge N, Naarding P, Koudstaal PJ. Hyperglicemia in the acute phase of stroke is not caused by stress. Stroke 1993; 24: 1129-32.

29. Soler S, Dávalos A, Ricart W, González-Huix F, Genís D. El perfil glucémico en el ictus, su relación con el estrés orgánico y con el pronóstico. Neurología 1991; 6: 400.

\title{
Tratamiento endovascular de fístulas arteriovenosas durales con drenaje venoso medular. Experiencia en 18 pacientes
}

\author{
J. Marey, R. Pego, G. Alonso, MS. López-Facal, M. Marín, A. Martínez a , JL. Díaz-Valiño a
}

\begin{abstract}
Resumen. Introducción y objetivos. Los autores presentan una serie de 18 pacientes con mielopatía en los que se diagnosticó una fístula dural arteriovenosa con drenaje venoso medular (FDdVM). El objetivo consistió en estudiar la efectividad inicial y a largo plazo de la embolización, como tratamiento inicial, utilizando partículas de polivinil-alcohol (PVA) y líquidos adhesivos, $N$-butil-cianoacrilato (NBCA). Material y métodos. Se obtuvieron imágenes de RM en todos los pacientes demostrando cambios en el parénquima medular compatibles con un proceso isquémico secundario a hipertensión venosa. Resultados. Todos los pacientes mejoraron inicialmente de su sintomatología y en algunos casos se demostró la desaparición de las imágenes previas en las RM de control. Se presentó recurrencia de la sintomatología y de la FDdVM angiográficamente demostrada en 8 casos (todos tratados con PVA). Estos pacientes fueron tratados con éxito mediante nueva embolización con NBCA. La embolización con PVA es ineficaz a largo plazo y no está exenta de riesgos. El tratamiento endovascular es menos invasivo, con menor morbilidad y asegura una rehabilitación más precoz que la cirugía. Si la embolización fracasa todavía puede ser tratado quirúrgicamente. Conclusiones. Recomendamos la embolización con NBCA como tratamiento inicial de elección en las FDdVM si contamos con una unidad de Neuroradiología intervencionista experimentada. Se precisa detenida evaluación clínica neurológica para establecer el diagnóstico de FDdVM. Finalmente recomendamos que los pacientes sean revisados estrecha y agresivamente. Son indispensables evaluaciones periódicas clínicas y radiológicas, incluyendo RM y angiografía medular para el tratamiento eficaz de estos pacientes [REV NEUROL 1998; 26: 793-9].
\end{abstract}

\section{Recibido: 14.01.97 Aceptado: 02.05.97}

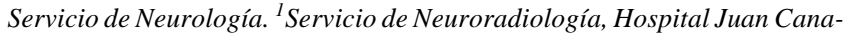
lejo. La Coruña. España.
Correspondencia: Dr. José Marey López. Santo Domingo, 4 - $4^{\circ}$. E-15001 La Coruña.

C 1998, REVISTA DE NEUROLOGÍA 
Palabras clave: Embolización. Fístula arteriovenosa. Médula espinal. Neuroradiología intervencionista.

\title{
ENDOVASCULAR TREATMENT OF DURAL ARTEROVENOUS FISTULAS BY MEDULLARY VENOUS DRAINAGE
}

\begin{abstract}
Summary: Introduction and objective. The authors report a series of 18 patients with myelopathy who were diagnosed of dural arteriovenous fistulas with venous medullary drainage (DFVMd). Purpose was to asses the effectiveness, initial and long term, of embolization, as the initial treatment, using polyvinyl alcohol particles (PVA) and liquid adhesives, $N$-butyl-cyanoacrylate (NBCA). Material and Methodes. Magnetic resonance images were obtained in all patients showing spinal cord tissue changes consistent with an isquemic process secondary to venous hypertension. All 18 patients showed initially an improvement in clinical symptoms, demonstrating previous MR images resolution. Results. The neurological status of 8 patients subsequently deteriorated with angiographically proven recurrences of their DFVMd. These patients underwent a second successful embolization procedure using NBCA. PVA embolization is long term ineffective and is not without risk. Endovascular treatment is less invasive than surgery, its morbidity is less, and it ensures earlier recovery for the patients. If embolization has failed, surgery can still be done. Conclusions. We recommend that NBCA embolization be the initial treatment of choice for DFVMd if referring the patient to an experienced interventional Neuroradiology unit is available. Careful clinical and neurologic examination is necessary to establish the diagnosis of DFVMd. Finally, we strongly recommend that patients be followed closely and aggressively. Periodic clinical and radiologic assessments, including MR and spinal angiography, are essential to achieve complete cure[REV NEUROL 1998; 26: 793-9].
\end{abstract}

Key words. Arteriovenous fistula. Embolization. Interventional Neuroradiology. Spinal cord.

\section{INTRODUCCIÓN}

Las malformaciones arteriovenosas espinales (MAVE) constituyen un grupo heterogéneo de anomalías vasculares. Estas lesiones han sido clasificadas en 4 tipos: Tipo I, fístulas durales con drenaje venoso medular, entre una rama dural del ramo espinal de una arteria radicular y una vena medular intradural; Tipo II, angiomas intramedulares; Tipo III, extensas malformaciones juveniles a menudo con extensión a estructuras paraespinales, y Tipo IV, fístulas arteriovenosas intradurales perimedulares, subclasificadas en IV a,b,c en función de su tamaño y número de aportes arteriales [1-4].

Las fístulas durales con drenaje venoso medular (FDdVM) son la anomalía vascular más frecuente de la patología raquimedular representando el $80 \%$ de las malformaciones vasculares espinales [5,6]. Se trata de fístulas arteriovenosas directas, probablemente adquiridas, de muy pequeño tamaño $(0,5-1,5 \mathrm{~mm}$ de diámetro), entre una arteria intercostal baja o una arteria lumbar, únicas en el $90 \%$ de los pacientes, y una vena, que se drena a contracorriente, de manera centrípeta hacia las venas perimedulares. Un segundo vaso está presente en el 10\% restante (de forma menos frecuente la fístula puede ser suplida por arterias sacras o ramas de las arterias ilíacas). Las venas, dilatadas, pueden drenar a niveles muy por encima de la localización del origen de la fístula con flujo característicamente lento. La arteria nutricia penetra en la fístula cerca de la salida dural de la raiz nerviosa. Es, pues, una fístula extramedular, de muy bajo flujo, situada en el espesor de la duramadre, asociada invariablemente a una alteración del drenaje venoso medular, no pudiéndose identificar el drenaje venoso epidural habitual $[4,7,8]$.

Las FDdVM causan el desarrollo de síntomas sensitivos y motores frecuentemente acompañados de disfunción esfinteriana y sexual de curso subagudo progresivo en pacientes, típicamente varones (más del 80\%), de mediana edad (50 a 70 años). El deterioro clínico lentamente progresivo es poco usual en la mayoría de procesos de etiología vascular, lo que produce frecuente retraso en la consideración de este diagnóstico. Una historia de varios meses desde la presentación clínica hasta la sospecha diagnóstica es habitual en pacientes con FDdVM, con el consiguiente empeoramiento pronóstico [4-6,9-13].

La fisiopatología responsable del lento desarrollo de la disfunción medular en las FDdVM parece relacionarse con hipertensión en las venas piales medulares y mielopatía subaguda necrotizante secundaria, idéntica a la descrita inicialmente por Foix y Alajoua- nine en 1926 [14]. Dado que no existe implicación del aporte arterial a la médula, no puede invocarse ningún mecanismo de 'robo' arterial para explicar el déficit neurológico en las FDdVM [16-19]. Recientemente, Hurst et al [15] han comunicado un caso angiográficamente confirmado de FDdVM con estudio patológico en el que mediante biopsia se confirma la congestión venosa e infarto medular como mecanismo responsable. La hipertensión transmitida a las venas intrínsecas medulares podría resultar en una reducción del gradiente de presión arteriovenosa medular con posterior disminución de la perfusión y progresiva hipoxia tisular. Pueden observarse vasodilatación intramedular y pérdida de capacidad autoreguladora como fenómenos asociados al edema medular. El resultado final es hipoxia-isquemia que conducen a la disfunción neurológica. A la elevada presión venosa intradural causada por la fístula puede contribuir una anomalía adquirida o del desarrollo del drenaje venoso intradural (la ausencia de defectos metaméricos asociados apuntan más a la existencia de un proceso adquirido) [20-23].

Tras la sospecha clínica, la primera exploración a realizar es el estudio con RM, siendo la angiografía espinal, en el presente, la única prueba diagnóstica que proporciona información suficiente para planificar el tratamiento.

La estrategia terapeútica consiste en reducir la presión venosa eliminando el shunt arteriovenoso. Existen dos alternativas: el abordaje quirúrgico basado en el clipaje de la vena de drenaje con coagulación-resección de la fístula, asociado frecuentemente a la necesidad de duroplastia a nivel de la lesión; y el tratamiento endovascular, tan efectivo como la cirugía si la zona fistulosa y el pie de la vena de drenaje son ocluidas permanentemente con el fin de evitar una revascularización rápida por anastomosis proveniente de arterias adyacentes $[4,8]$. Si contamos con un Servicio de Neuroradiología intervencionista experimentado, la embolización es el tratamiento de elección.

Comunicamos nuestra experiencia en 18 pacientes con FDdVM tratados inicialmente con embolización endovascular.

\section{MATERIAL Y MÉTODOS}

Hemos revisado retrospectivamente una serie de 18 pacientes consecutivos con FDdVM diagnosticados mediante angiografía entre el año 1986 y el 1994, todos ellos varones con una edad comprendida entre los 48 y 69 años (media de 61,5 años). El período de seguimiento medio fue de 4,5 años.

Tras realizar historia clínica y exploración neurológica completa, se obtuvieron muestras para la determinación de hemograma, bioquímica habi- 


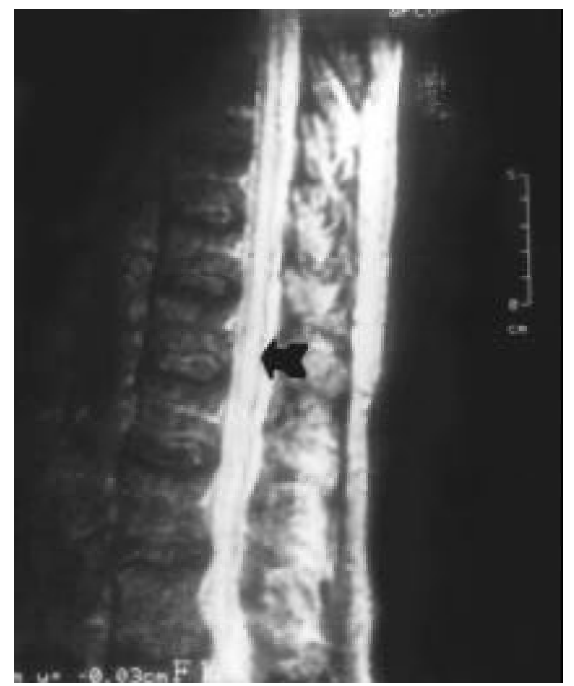

Figura 1. Caso 17. RM medular dorsal. Secuencia sagital en $T_{2}$. Hiperseñal centromedular (flecha), compatible con un proceso isquémico.

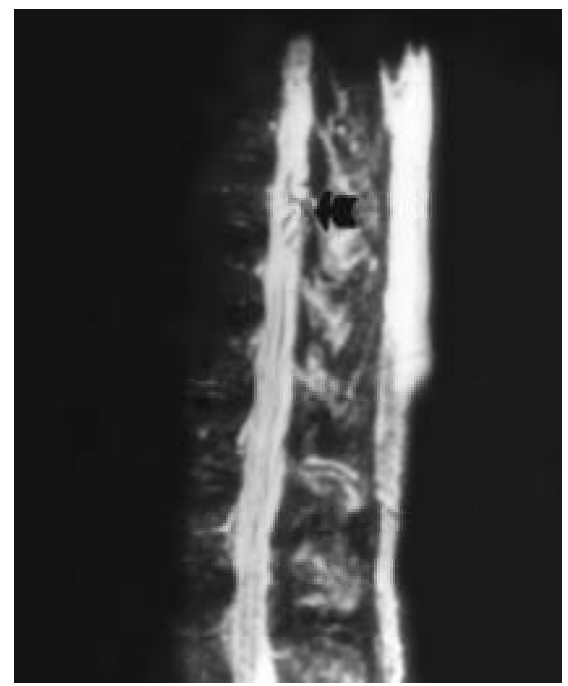

Figura 2. Caso 17. RM medular dorsal. Secuencia sagital en $\mathrm{T}_{2}$. Pequeñas hiperintensidades sugestivas de estructuras venosas dilatadas (flecha).

La clínica registrada en nuestros enfermos fue la habitual en las FDdVM: paraparesia aislada progresiva, frecuentemente exacerbada por el ejercicio (12 pacientes), siendo más común el inicio bilateral asimétrico de la clínica. La pérdida brusca de fuerza con el ejercicio en miembros inferiores seguida de paraparesia progresiva fue el modo de presentación solamente en dos casos. La sintomatología sensitiva aislada (parestesias, entumecimiento, radiculalgia $\mathrm{L}_{4}, \mathrm{~L}_{5} \mathrm{O} \mathrm{S}_{1}$, cruralgia, dorsalgia) como debut se registró en tres pacientes y en uno el primer síntoma fue incontinencia urinaria. Todos los pacientes presentaron síntomas motores (paraparesia, claudicación medular de la marcha) y sensitivos, en algún momento del cuadro clínico. Siete enfermos presentaron disfunción urinaria y rectal (incontinencia, retención urinaria, disminución del tono rectal); en ninguno pudo

tuales y otras: test de función tiroidea, espectro electroforético de proteínas séricas, determinación de $\mathrm{B}_{12}$ y ácido fólico, VSG, ANA y titulación Lyme. No se realizó de forma sistemática punción lumbar en nuestros enfermos, dado que no aporta datos de importancia para el diagnóstico y se han comunicado empeoramientos clínicos pospunción. En todos los casos se realizó RM medular con una unidad 0,5-T con cortes sagitales y axiales, como primer paso del protocolo diagnóstico. En tres pacientes se practicó mielografía dorsolumbar con medios de contraste hidrosolubles y el paciente en posiciones prono y supino.

El diagnóstico radiológico se realizó siempre mediante angiografía medular selectiva con imágenes convencionales y de substracción digital, realizada cuando el cuadro clínico y los hallazgos de la RM eran sugestivos o simplemente sospechosos de la presencia de una malformación vascular medular. No se precisó anestesia general en la mayoría de los casos. La angiografía identifica el lugar exacto de la comunicación arteriovenosa y las características del drenaje venoso. Se localiza también la arteria espinal anterior y su posible relación con la arteria aferente de la fístula. El estudio angiográfico y embolización se realizó, en la misma intervención, mediante abordaje transfemoral que permite una visión general de todas las arterias dorsolumbosacras y frecuentemente la arteria de Adamkiewicz, cuyo origen en relación al de la fístula debe de ser tenido en cuenta en el momento de decidir la estrategia terapéutica. Se prosigue con cateterización selectiva de las arterias intercostales lumbares o sacras que dan origen a las arterias durales que suplen la FDdVM.

Dos condiciones deben ser establecidas antes del inicio del tratamiento: la posibilidad y plan de abordaje de un cateterismo superselectivo estable de la arteria nutricia, que permita una embolización endovascular eficaz; y excluir la existencia de anastomosis entre esta arteria y la espinal anterior. El material de embolización utilizado consistía en partículas de PVA (polivinil-alcohol) (100 - 500 $\mu \mathrm{m}$ ) en 10 pacientes de los primeros años de la serie y líquidos adhesivos, N-butil-cianoacrilato (NBCA) en los 8 restantes y en las recurrencias. Se realizó control clínico, RM y, cuando fue preciso, angiográfico, en períodos de 3, 6 meses y 1 año postembolización.

\section{RESULTADOS}

El cuadro clínico en nuestra serie de 18 varones, entre 48 y 69 años, fue el de mielopatía dorsolumbar de comienzo progresivo (duración media de la sintomatología desde el inicio al diagnóstico de 2 meses (15 días - 11 meses)). No existían antecedentes personales o familiares de enfermedad neurológica, ni traumatismos previos al comienzo de la debilidad, aunque sí se recogen caídas posteriores a la instauración de la pérdida de fuerza. identificarse alteración de la función sexual atribuible exclusivamente al inicio de la FDdVM.

Independientemente de la forma de comienzo, todos evolucionaron hacia una afectación sensitivomotora y esfinteriana en diversos niveles de gravedad (según el tiempo de evolución hasta el tratamiento): un cuadro de radiculomielopatía dorsolumbar que a la exploración neurológica inicial se identificaba por orden de frecuencia con síndrome de cono medular, cola de caballo y paraparesia aislada. El factor agravante más frecuentemente identificado fue el ejercicio (caminar), seguido de posición sentada prolongada.

No se hallaron alteraciones relevantes en estudios bioquímicos ni hematológicos.

La RM medular fue la primera prueba diagnóstica realizada. En la secuencia sagital y axial ponderada en $\mathrm{T}_{1}$ los hallazgos más frecuentes fueron aumento de volumen e hiposeñal del cono medular e hiposeñal centromedular. Es preciso destacar la alta incidencia (14 casos) de canal lumbar estrecho. En la secuencia $\mathrm{T}_{2}$ destaca hiperseñal centromedular (médula dorsal y cono) y pequeñas hiperseñales sugestivas de estructuras venosas dilatadas (Figs. 1 y 2).

Es importante señalar dos aspectos de los hallazgos RM en nuestros pacientes: 1. Las alteraciones descritas se extendían en la mayoría de los casos (16 pacientes) por encima del nivel de alteración que indicaba la exploración clínica, y 2. En dos casos, los hallazgos poco significativos en la RM ya que no permitieron valorar adecuadamente la posibilidad de FDdVM.

En los tres casos en que se realizó mielografía dorsolumbar, entre los que se incluyen los dos con RM poco significativa, se evidenciaron imágenes vasculares serpiginosas a nivel de la médula que sugerían la presencia de una malformación vascular y que generalmente permitían excluir una variante normal.

La angiografía medular fue diagnóstica en todos los casos. La inyección selectiva de la arteria aferente implicada da como resultado el relleno precoz de las venas piales medulares. El pequeño tamaño de la FDdVM puede causar que ésta resulte de dificultosa identificación angiográfica, por ello, el relleno precoz de las venas piales es el dato patognomónico que detecta la posible existencia de una FDdVM (Fig. 3). 


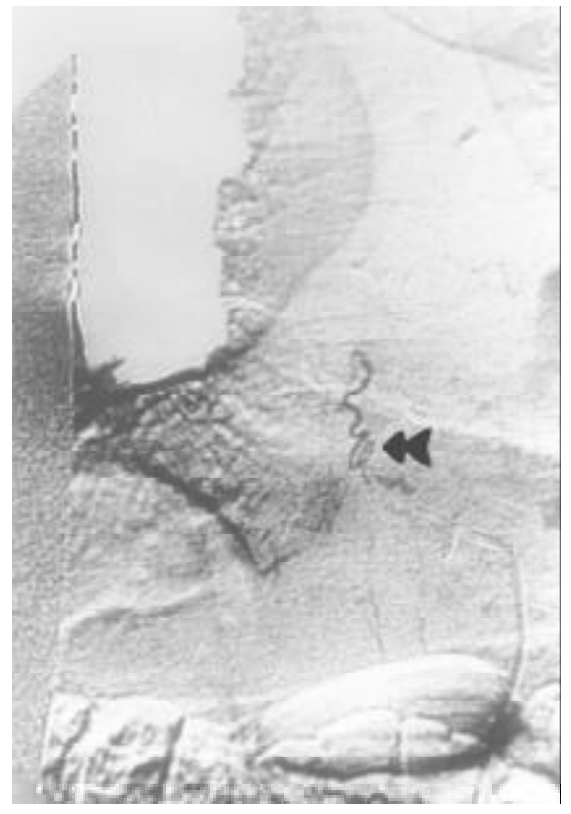

Figura 3. Caso 17. Cateterismo selectivo de la 9a arteria intercostal izquierda mostrando la localización de la fístula (flecha) y el drenaje venoso.
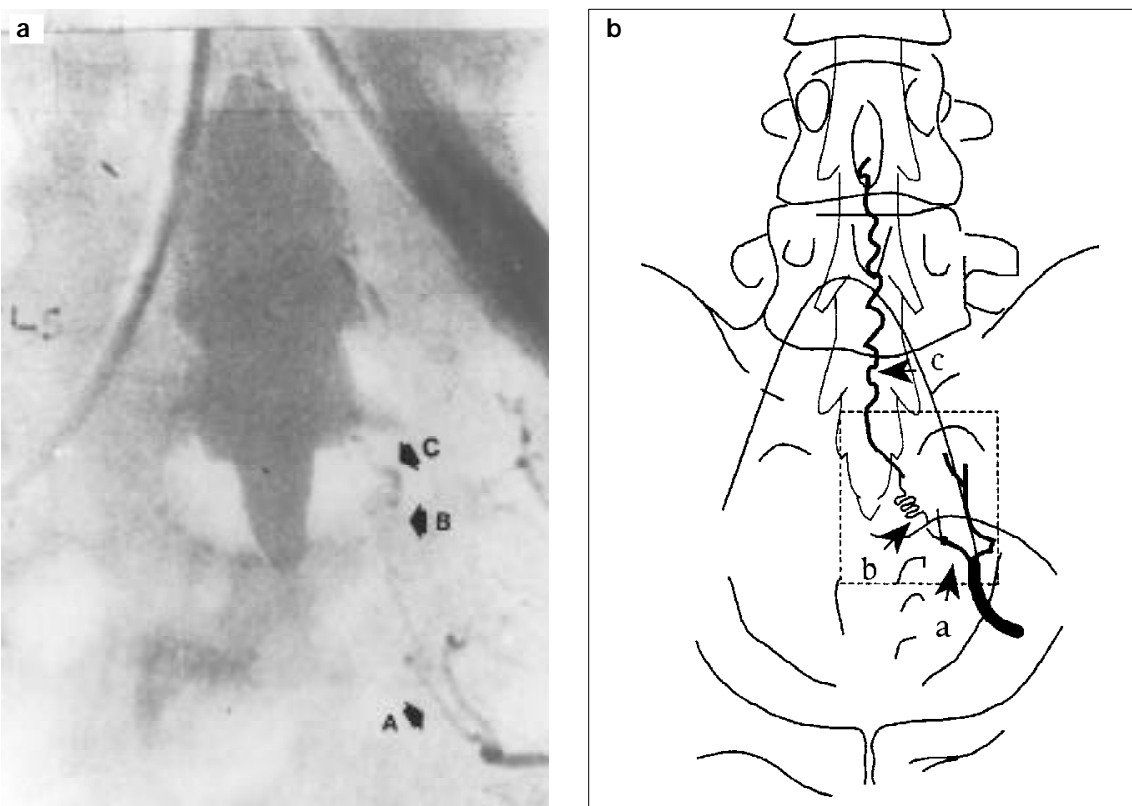

Figura 4. a) Caso 6. Cateterismo de la arteria ilíaca interna izquierda que muestra la arteria sacra media (a), la fístula (b) e inicio de la vena de drenaje (c); b) Esquema correspondiente a la figura 4a que muestra las mismas estructuras.
La fístula era única en todos los casos, con localización en región torácica alta $\left(\mathrm{T}_{5}\right.$ a $\left.\mathrm{T}_{8}\right)$ en 6 casos, torácica baja $\left(\mathrm{T}_{9}\right.$ a $\left.\mathrm{T}_{12}\right)$ en 9 , lumbar en 2 y sacra en 1 . Las arterias aferentes eran las intercostales, las arterias lumbares y, en un caso, la arteria sacra media (Figs. 4a y 4b). La vena eferente única, dilatada, presentó en la mayoría de los casos trayecto ascendente intradural con drenaje anormal en el sistema venoso perimedular. El retorno venoso de la arteria espinal anterior no era visible incluso en $60 \mathrm{~s}$ (normal 15-25s); en este sentido, la demostración de una fase venosa normal cuando se considera el diagnóstico de FDdVM descarta prácticamente su existencia. La angiografía postembolización demostró oclusión completa de la zona fistulosa en los 18 casos, (Fig. 5).

Tras la embolización todos los pacientes mejoraron clínicamente. La sintomatología que de forma más espectacular mejoró desde los primeros días fue la motora, aunque nunca hasta su resolución completa. Los problemas sensitivos tardaron más tiempo en subsanarse, sobre todo en el caso de las parestesias, aunque desaparecieron en su totalidad en el $50 \%$ de los pacientes a lo largo de la evolución. La clínica esfinteriana no remitió al cien por cien en ninguno de ellos precisándose estudios urodinámicos y cateterismos vesicales intermitentes prolongados. Los pacientes que más precozmente y con mayor intensidad presentaron alivio sintomático fueron aquéllos en los que el período previo al diagnóstico y tratamiento fue menor.

En la RM de control postembolización y en imágenes ponderadas en $\mathrm{T}_{1}$ destacaban zonas de atrofia medular y en las secuencias $\mathrm{T}_{2}$ reducción tanto de la hiperseñal centromedular como de las imágenes serpiginosas vasculares, en 14 de los 18 casos (Fig. 6).

En ocho casos, todos embolizados con PVA, se produjo recurrencia de la clínica por repermeabilización de la fístula (acudiendo sobre todo por la recidiva motora) en un tiempo medio de 8 meses (2-25 meses) siendo tratados con nueva embolización (NBCA), exitosa angiográfica y clínicamente en todos ellos hasta el momento actual.

\section{DISCUSIÓN \\ Revisión de la literatura}

La existencia de malformaciones vasculares del canal espinal (MAVE) con aporte arterial desde arterias durales y drenaje venoso medular fue establecida como entidad clinico-patológica en 1977 por Kendall y Logue [18]. La incidencia real de las FDdVM, también denominadas MAVE tipo I, es poco conocida; en tres series amplias de MAVE la proporción de FDdVM oscila entre el $30 \%$ y el $95 \%,[5,6,10]$. La mayoría de los autores las consideran una lesión adquirida [23,24].

El paciente tipo es un varón de mediana edad con una mieloradiculopatía de lenta instauración y progresión, a menudo agravada por la actividad física [4-6,9-13]. Estas lesiones se localizan más frecuentemente en región dorsal baja, respetando típicamente los miembros superiores [7,25]. Tales características clínicas varían de forma significativa de las que presentan las MAVE intramedulares (pacientes más jóvenes, de ambos sexos, con presentación aguda, frecuente hematomielia o hemorragia subaracnoidea, sin localización predilecta en el canal espinal) $[4,5,11,12,26]$. Se han comunicado fístulas con localizaciones del nido fistuloso intracraneales y en la región sacra con drenaje venoso medular, como en el caso de uno de nuestros pacientes con aporte a través de la arteria sacra media [7,25] (Fig. 4a).

\section{Fisiopatología}

Los estudios histopatológicos en diversas series de FDdVM muestran que la localización del shunt es transdural y típicamente único, con un nido constituido por una red de colaterales de arterias durales que forman el aporte arterial. El drenaje venoso es de localización intradural; su aspecto más distrófico que displásico es un argumento a favor de un origen adquirido de estas lesiones $[23,24]$. Otros razonamientos en pro de su etiología adquirida son: 1. La existencia de comunicaciones arteriovenosas potenciales en la duramadre normal tanto craneal como espinal $[4,11,18,19,27] ; 2$. La edad de presentación tardía; 3 . Su bajo 


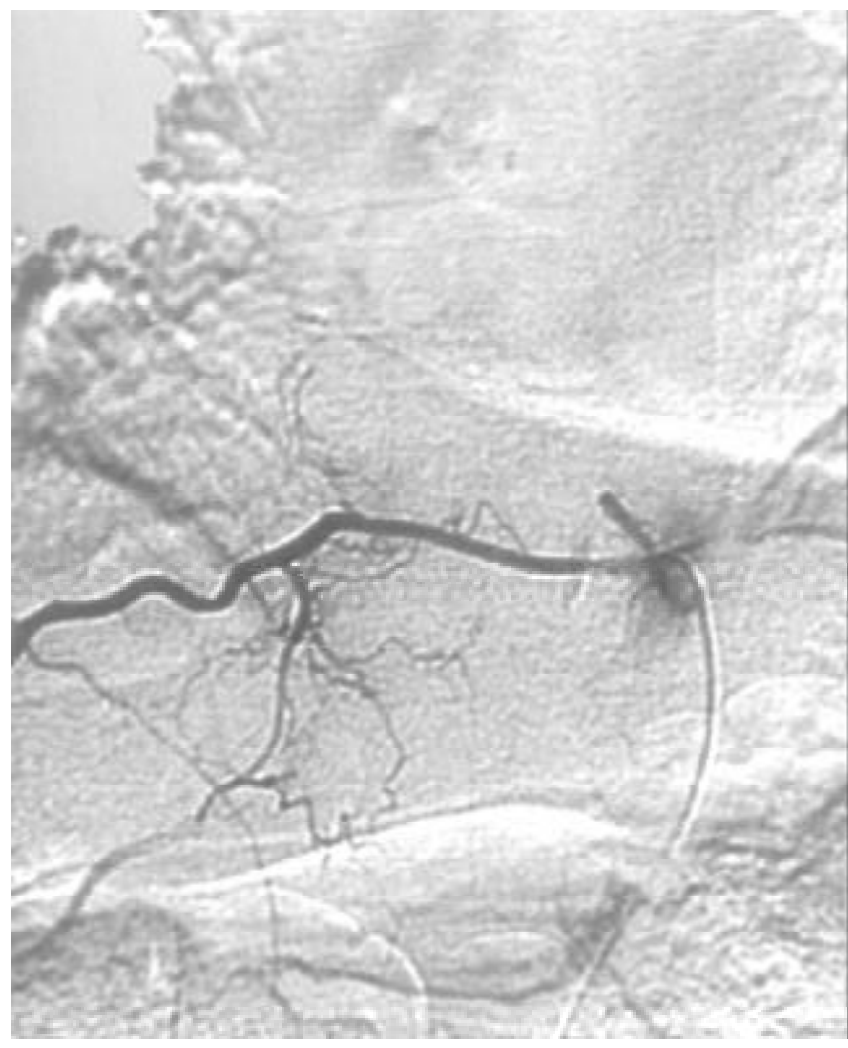

Figura 5. Caso 17. Control postembolización con NBCA de la fístula mos trada en la figura 3.

flujo con ausencia de drenaje hacia el plexo venoso epidural, y 4. La similitud con las fístulas durales craneales, también consideradas de causa adquirida [28].

El mecanismo fisiopatológico generalmente aceptado en las FDdVM, ya propuesto por Aminoff et al [17] en 1974, se basan en el aumento de presión venosa resultante de las anastomosis entre la vena de drenaje y el plexo venoso coronal que realiza el drenaje normal de la médula. Esta hipertensión venosa resulta en una disminución de la perfusión del parénquima medular en las regiones donde el aporte arterial es más débil y el plexo venoso coronal está más desarrollado, como usualmente sucede en la unión toracolumbar [29]. En este sentido Merland et al [10] mediante angiografía selectiva en pacientes con FDdVM interpretaron la ausencia de drenaje venoso radicular normal como un signo directo de hipertensión venosa. Si esta ausencia de drenaje es adquirida o congénita es un dato desconocido pero que parece no normalizarse en el seguimiento angiográfico de pacientes tratados con éxito.

Coexisten, por tanto, dos problemas: la congestión venosa resultante de la fístula y un pobre drenaje medular a través de las venas radiculares. Se han realizado estudios hemodinámicos de las FDdVM, entre los que destaca el realizado por Hassler [30], que confirmó la naturaleza de bajo flujo y elevación de presión en la vena arterializada proporcional a la presión arterial, postulando que esta relación directa podría ser la causante del empeoramiento de los síntomas durante el ejercicio.

Varios estudios $[18,32,33]$ han sugerido que un canal espinal estrecho (compresión crónica de venas radiculares por hipertrofias oseas y ligamentosas) puede empeorar la hipertensión venosa, asociación que se ha visto confirmada en nuestra serie (14 de

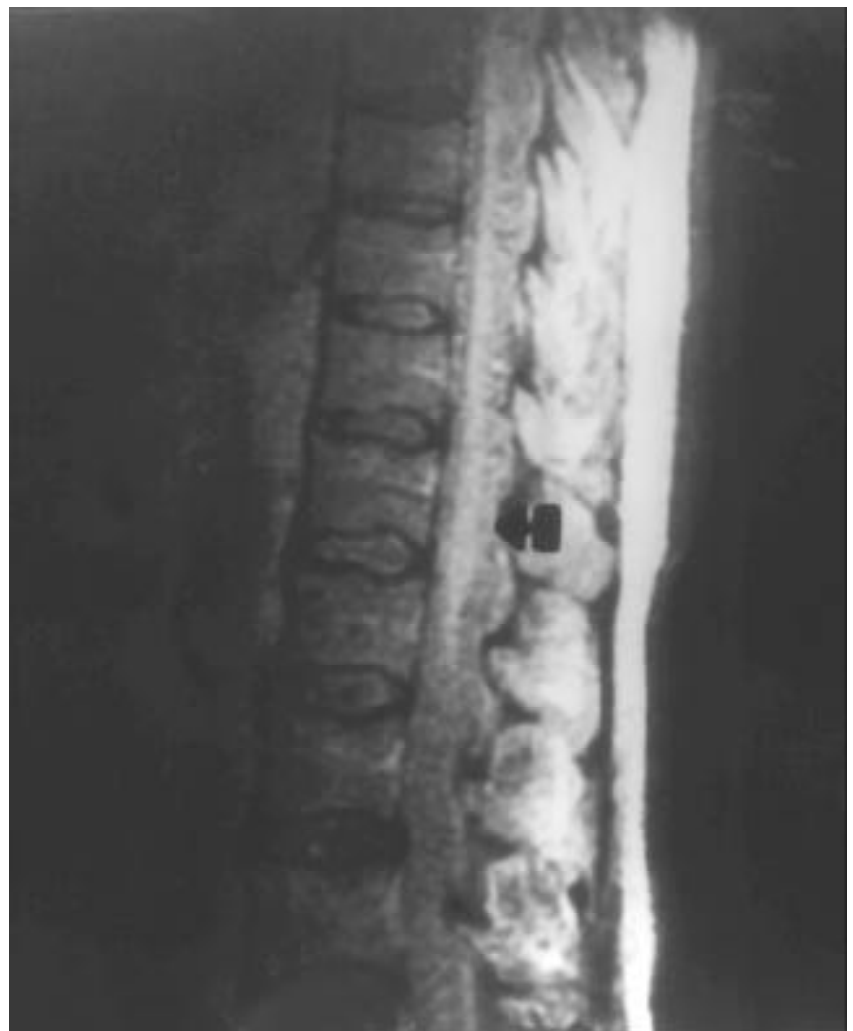

Figura 6. Caso 17. RM medular dorsal de control, realizada a los 3 meses de la embolización con NBCA. Secuencia sagital en $T_{2}$. Desaparición de la hiperseñal previa (flecha).

18 enfermos presentaban hallazgos de estenosis crónica de canal) aunque existe consenso general en cuanto a que son dos entidades probablemente no relacionadas etiológicamente.

\section{Estudios diagnósticos}

La RM ha proporcionado un medio para documentar los cambios isquémicos medulares causados por una FDdVM y, lo que es mucho más importante, su resolución tras el cierre de la fístula, constituyéndose así en una exploración de valor pronóstico (Figs. 1,2 y 6). Varios autores han resaltado la visualización del plexo venoso dilatado en la superficie dorsal medular como un hallazgo característico de la presencia de un shunt arteriovenoso asociado a la existencia de hiperseñales medulares en $\mathrm{T}_{2}$ [34-38]. No obstante, debe destacarse que en ningún caso son hallazgos específicos (el diagnóstico diferencial de la neuroimagen incluye: tumor intramedular, inflamación, desmielinización, isquemia de origen arterial o más raramente infarto debido a trombosis venosa) así como que una RM normal no excluye la presencia de una FDdVM.

La presencia de hallazgos típicos de FDdVM en la RM hace precisa la realización de una angiografía, única prueba diagnóstica.

En cuanto a la sensibilidad comparada entre RM y mielografía como técnicas de screening previas a la angiografía existen discrepancias en la literatura [34-39]. En general, nosotros preferimos no realizar mielografía en el caso de que la RM sea altamente sugestiva de la presencia de una malformación vascular espinal, dado que es una técnica más invasiva con similar rendimiento.

La angiografía es el único medio real de identificar la localización exacta del nido fistuloso, punto fundamental sea cual sea 
la estrategia terapeútica que vaya a elegirse, embolización o neurocirugía. Nosotros iniciamos la exploración angiográfica mediante inyección bifemoral retrógrada. Cuando la arteria de Adamkiewicz es identificada se obtienen varias tomas en tiempos tardíos. La imposibilidad para visualizar las venas radiculares normales sugiere la presencia de hipertensión venosa en relación con una FDdVM que suele ser fácilmente identificable aunque tenga localización sacra gracias a la técnica retrógrada bifemoral.

Si la angiografía es negativa, pero las imágenes por RM o mielografía son altamente características, es preciso estudiar el sistema vertebrobasilar y el carotídeo ya que existen fístulas intracraneales que poseen drenaje venoso preferente medular y por ello se presentan con clínica medular [4].

La angiografía medular nos proporciona, por tanto: localización exacta de la fístula, tamaño, aferencias arteriales (número, tipo, relación con la circulación normal medular), drenajes venosos (número, situación, dirección y longitud hasta la médula) y la posibilidad, en su caso, de iniciar la embolización de la lesión en la misma intervención $[6,40,41,42]$.

\section{Tratamiento}

Hemos revisado retrospectivamente las historias y hallazgos angiográficos de 18 pacientes con FDdVM embolizadas como primer tratamiento tanto con PVA como con NBCA. En total se realizaron 26 procedimientos en estos pacientes. Se constataron recurrencias angiográficamente en 8 de los 10 pacientes tratados con PVA.

Diferentes autores proponen la cirugía como tratamiento electivo $[6,19,43]$, con mejoría de la sintomatología previamente progresiva en un número de pacientes significativo. Otros proponen la embolización como el primer tratamiento a realizar en estos enfermos [44-46].

Las FDdVM pueden ser tratadas con éxito únicamente con la oclusión permanente del nido fistuloso y el pie de la vena de drenaje. Aunque la angiografía de control postembolización con partículas de PVA muestre una oclusión inicial perfecta, en la mayoría de los casos la localización de la oclusión es proximal a la fístula y se produce recanalización de la arteria aferente o reclutamiento de colaterales inicialmente no evidentes que producen la recurrencia de la FDdVM [44].

El tamaño de la fístula varía en diámetro entre 0,5-1,5 mm en nuestra serie, este tamaño puede resultar suficiente para permitir el paso de partículas hacia la vena de drenaje. El problema reside en que es muy difícil utilizar una partícula del tamaño exacto de la fístula, ni demasiado grande que no alcance ésta y ocluya la arteria aferente, ni demasiado pequeña que pase a través de la vena de drenaje hacia el sistema venoso. El paso de material de embolización a través de la fístula hacia el sistema venoso puede comprometer el ya de por sí precario flujo venoso medular, empeorando la mielopatía. En resumen: oclusión proximal de la arteria aferente, recanalización, desarrollo de colaterales previamente no identificables y el paso de las partículas menores hacia el sistema venoso son las razones principales para desaconsejar la utilización de PVA en las FDdVM y que explican las recurrencias observadas en nuestra serie y en las de otros autores [43,44,46-48].

Las arterias que suplen la FDdVM son de tamaño menor que el nido fistuloso en sí; por tanto, para obtener una oclusión eficaz, es necesario un material que sea capaz de alcanzar un shunt de tamaño considerable a través de pequeñas arterias. Además es preciso evitar la formación de colaterales, que vuelvan a alimentar la fístula, mediante la oclusión del pie de la vena de drenaje.
La embolización de las FDdVM con NBCA cumple estas necesidades y permite el cierre permanente de estas lesiones. Una de las complicaciones del tratamiento con adhesivos es la polimerización del producto en la arteria aferente, que no ocurrió en nuestra serie pero sí ha sido comunicada $[7,10,44,48]$. En este caso es preciso realizar cirugía para cerrar la vena de drenaje. Una contraindicación de la embolización con NBCA, aparte de las descritas (arteria espinal anterior con origen común a la arteria dural que suple la fístula en la misma arteria intercostal y la imposibilidad de cateterización superselectiva estable), es la localización de la fístula en región torácica alta. En esta zona la vena de drenaje tiene un recorrido muy corto antes de unirse al sistema venoso medular, presentando un margen de riesgo demasiado alto para la polimerización del producto en ese sistema venoso.

Nosotros recomendamos la embolización de las FDdVMcomo tratamiento de elección inicial, siempre que se disponga de un Servicio de Neuroradiología experimentado. Los porcentajes de éxito-complicaciones son comparables a la cirugía en series amplias de la literatura [10,44-46]. La embolización con NBCA es una técnica menos invasiva y permite una recuperación más precoz del enfermo dada la posibilidad de iniciar un programa de rehabilitación precoz el mismo día después de la embolización dado que es una técnica realizada sin demoras en el mismo tiempo de la angiografía diagnóstica. Si fracasa, es posible realizar cirugía en una segunda intervención como alternativa.

En nuestra opinión, la cirugía, como técnica inicial, debe reservarse para los casos de implicación de la arteria espinal anterior o imposibilidad de cateterismo estable, como ya hemos descrito. La cirugía es también la técnica a utilizar en el caso de recidiva de la fístula en un paciente embolizado previamente con adhesivos. En el caso de que el material inicial hubiera sido partículas de PVA creemos indicado intentar una segunda embolización con NBCA.

La aparición de un nuevo producto no reabsorbible, las emboesferas, con excelente resultado en las fístulas intracraneales, promete buenas posibilidades para la embolización de las FDdVM.

\section{CONCLUSIONES}

Es muy importante señalar que sólo una alta sospecha clínica y cuidadosa exploración neurológica permiten establecer el diagnóstico precoz de una patología frecuente, que suele presentarse como una mielopatía subaguda lentamente progresiva (la etiología vascular suele considerarse en procesos de presentación brusca y curso más agudo), cuyo pronóstico está en función directa del momento en que se inicie el tratamiento.

Es habitual que estos pacientes sean evaluados en primer lugar por estenosis de canal lumbar, con frecuencia coexistente, pero clínicamente poco significativa, e incluso son sometidos a procedimientos quirúrgicos inapropiados y potencialmente yatrogénicos.

Tras la imprescindible sospecha diagnóstica, debe iniciarse precozmente la evaluación complementaria con RM, mielografía (sólo en el caso de que las imágenes de resonancia no sean sugestivas) y angiografía medular.

Finalmente, dada nuestra experiencia, queremos recomendar que estos pacientes sean revisados estrecha y agresivamente, incluyendo evaluaciones periódicas clínicas y radiológicas que incluyan RM y, si es preciso, angiografía. 


\section{BIBLIOGRAFIA}

1. Anson JA, Spetzler RF. Classification of spinal arteriovenous malformations and implications for treatment. Barrow Neurol Inst Q 1992; 8: 2-8.

2. Anson JA, Spetzler RF. Spinal dural arteriovenous malformations. In Awad IA, Barrow DL, eds. Dural arteriovenous malformations, Park Ridge, III: American Association of Neurological Surgeons, 1993: 175-91.

3. Malis LI. Arteriovenous malformations of the spinal cord. In Youmans JR, eds. Neurological Surgery. Vol. 3. 2 ed. Philadelphia: WB Saunders; 1982. p. 1850-74.

4. Guimaraens L, Vázquez-Añón V. Fístulas arteriovenosas durales raquídeas con drenaje venoso medular. En Rubio F, Sancho-Rieger J, eds. Patología vascular medular. Barcelona: Editorial MCR; 1993 p. 129-42.

5. Rosenblum B, Oldfield EH, Doppman JL, et al. Spinal arteriovenous malformations: A comparison of dural arteriovenous fistulas and intradural AVM's in 81 patients. J Neurosurg 1987; 67: 795-802.

6. Symon L, Kuyama H, Kendall B. Dural arteriovenous malformations of the spine. Clinical features and surgical results in 55 cases. J Neurosurg 1984; 60: 238-47.

7. Partington MD, Rüfenacht DA, Marsh WR, Piepgras DG. Cranial and sacral dural fistulas as a cause of mielopathy. J Neurosurg 1992; 76: 615-22.

8. Deen HG, Kent MD, Nelson MD, Gonzales GR. Spinal dural arteriovenous fistula causing progressive myelopathy: Clinical and imaging considerations. Mayo Clin Proc 1994; 69: 83-84.

9. Logue V. Angiomas of the spinal cord: Review of the pathogenesis, clinical features and result of surgery. J Neurol Neurosurg Psychiatry 1979; 42: 1-11.

10. Merland JJ, Assouline E, Rüfenacht D et al. Dural spinal arteriovenous fistulae draining into medullary veins. Clinical and radiological results of treatment (embolization and surgery) in 56 cases. In Valk J, ed. Neuroradiology 1985/1986. Amsterdam: Elsevier; 1986. p. 283-9.

11. Oldfield EH, Doppman JL. Spinal arteriovenous malformations. Clin Neurosurg 1988; 34: 161-83.

12. Cogen P, Stein BM. Spinal cord arteriovenous malformations with significant intramedullary components. J Neurosurg 1983; 59: 471-8

13. Tobin WD, Layton DD. The diagnosis and natural history of spinal cord arteriovenous malformations. Mayo Clin Proc 1976; 51: 637.

14. Foix C, Alajouanine T. La myélite nécrotique subaigué: myélite centrale angéiohypertrophique a évolution progressive. Rev Neurol (Paris) $1926 ; 33: 1-42$

15. Hurst RW, Kenyon LC, Lavi E, et al. Spinal dural arteriovenous fistula: The pathology of venous hypertensive myelopathy. Neurology 1995 45: $1309-13$.

16. Kim R. Necrotizing myelopathy. AJNR Am J Neuroradiol 1991; 12: 1084-6.

17. Aminoff M, Logue V. The prognosis of patients with spinal vascular malformations. Brain 1974; 97: 211-8.

18. Kendall B, Logue V. Spinal epidural angiomatous malformation draining into intrathecal veins. Neuroradiology 1977; 13: 181-9.

19. Oldfield E, DiChiro G, Quindlen E, et al. Successful treatment of a group of spinal cord arteriovenous malformations by interruption of dural fistula. J Neurosurg 1983; 59: 1019-30.

20. Criscuolo G, Oldfield E, Doppman J. Reversible acute and subacute myelopathy in patients with dural arteriovenous fistulas. J Neurosurg 1989; 70: 354-9.

21. Adams J, Duchen L. Greenfield's Neuropathology. New York: Oxford University Press; 1992. p. 1107-10.

22. Mirich D, Kucharczyk W, Keller M, Deck J. Subacute necrotizing myelopathy. MR imaging in four pathologically proved cases. AJNR Am J Neuroradiol 1991; 12: 1077-83.
23. Benhaiem N, Poirier J, Hurth M. Arteriovenous fistulae of the meninges draining into the spinal veins. A histological study of 28 cases. Acta Neuropathol 1983; 62: 103-1.

24. Cahan LD, Higashida RT, Halbach VV, et al. Variants of radiculomeningeal vascular malformations of the spine. J Neurosurg 1987; 66: 333-7.

25. Burguet JL, Dietemann JL, Wackenheim A, et al. Sacral meningeal arteriovenous fistula fed by branches of the hypogastric arteries and drained through medullary veins. Neuroradiology 1985; 27: 232-7.

26. Odom GL. Vascular lesions of the spinal cord: Malformations, spinal subarachnoid and extradural hemorraghe. Clin Neurosurg 1962; 8: 196-236.

27. Leslie D, Chan M, Randall T, et al. Variants of radiculomeningeal vascular malformations of the spine. J Neurosurg 1987; 66: 333-7.

28. Douglas A, Graeb M, Clarisse L, Dolman M. Radiological and pathological aspects of dural arteriovenous fistulas. Case report. J Neurosurg 1986; 64: 962-7.

29. Gillilan LA. Veins of the spinal cord. Anatomic details: Suggested clinical applications. Neurology 1970; 20: 860-8.

30. Hassler W, Thron A, Grote EH. Hemodynamics of spinal dural arteriovenous fistulas. An intraoperative study. J Neurosurg 1975; 70: 360-70.

32. Madsen JR, Heros RC. Spinal arteriovenouss malformations and neurogenic claudication. Report of two cases. J Neurosurg 1988; 68: 793-7.

33. Aminoff MJ, Barnard RO, Logue V. The pathophysiology of spinal vascular malformations. J Neurol Sci 1974; 23; 255-63.

34. Isu T, Iwasaki Y, Akino M, et al. Magnetic resonance imaging in cases of spinal dural arteriovenous fistula. Neurosurgery 1989; 24: 919-23.

35. Masaryk T, Ross J, Modie M, et al. Radiculomeningeal vascular malformations of the spine: MR imaging. Radiology 1987; 164: 845-9.

36. Larsson E, Desai P, Hardin C, et al. Venous infarction of the spinal cord resulting from dural arteriovenous fistula: MR imaging findings. AJNR Am J Neuroradiol 1991; 12: 739-43.

37. Doppman JL, Di Chiro G, Dwyer AJ, et al. Magnetic resonance imaging of spinal arteriovenous malformations. J Neurosurg 1987; 66: 830-4.

38. Minami S, Sagoh T, Nishimura K, et al. Spinal arteriovenous malformation: MR imaging. Radiology 1988; 169: 109-15.

39. Assouline E, Gelbert F, Dormont D, et al. MRI study of dural arteriovenous fistulas draining into the external spinal veins. Seven cases. J Neuroradiol 1988; 15: 1-12.

40. Melki JP, Riche MC, Reizine D, et al. Simultaneous bifemoral retrograde arteriography under pressure. First stage in angiographic exploration of the spine and spinal cord. J Neuroradiol 1986; 13: 62-70.

41. Willinsky R, Lasjaunias P, Terbrugge K, Hurth M. Angiography in the investigation of spinal dural arteriovenous fistula. A protocol with application of the venous phase. Neuroradiology 1990; 32: 114-6.

42. Choi IS, Berenstein A. Surgical neuroangiography of the spine and spinal cord. Radiol Clin North Am 1988; 26: 1131-41.

43. Morgan MK, Marsh WR. Management of spinal dural arteriovenous malformations. J Neurosurg 1989; 70: 832-6.

44. Nichols DA, Rufenacht D, Clifford R, Forbes G. Embolization of spinal dural arteriovenous fistula with Polyvinyl Alcohol Particles: Experience in 14 patients. AJNR 1992; 13: 933-40.

45. Scialfa G, Scotti G, Biondi A, De Grandl C. Embolization of vascular malformations of the spinal cord. J Neurosurg Sci 1985; 29: 1-9.

46. Choi IS. Spinal dural arteriovenous fistula: The role of PVA embolization. AJNR 1992; 13: 941-2.

47. Hall WA, Oldfield EH, Doppman JL. Recanalization of spinal arteriovenous malformations following embolization. J Neurosurg 1981; 70: 714-20.

48. Barth MO, Chiras J, Rose M, et al. Résultats de 1'embolisation des fístules artério-veineuses durals rachidiennes à drainage veineaux pèrimédullaire. Neurochirurgie 1984; 30: 381-6.

\section{Comentario}

\section{F. Rubio}

Las fístulas arteriovenosas durales con drenaje venoso medular constituyen un grupo bien diferenciado dentro de las MAV de la

Recibido: 14.04.97. Aceptado: 02.05.97.

Servicio de Neurología. Ciudad Sanitaria y Universitaria de Bellvitge. Universidad de Barcelona. Barcelona, España. médula tanto por los aspectos fisiopatológicos como por las consideraciones terapéuticas. La descripción de Merland en 1980 [1]

Correspondencia: Dr. Francisco Rubio. Servicio de Neurología. Ciudad Sanitaria y Universitaria de Bellvitge. Feixa Llarga, s/n. E-08907 L'Hospitalet de Ll., Barcelona.

(C) 1998, REVISTA DE NEUROLOGÍA 
debe considerarse como maestra al describir aspectos no conocidos, ya que, aparte de la ausencia de nidus angiomatoso, se observa un llenado retrógrado de la vena de drenaje con aumento de la presión y alteración medular.

El trabajo de Marey et al constituye un ejemplo evidente de las posibilidades y el desarrollo de las prácticas intervencionistas en nuestro país como alternativa a los tratamientos quirúrgicos empleados hasta ahora.

Desde nuestro punto de vista, algunos problemas deben ser planteados en los casos de fístulas durales si queremos avanzar en la mejoría de estos pacientes:

- Dada la edad habitual de presentación, debe considerarse que estos casos son adquiridos y no tenemos una idea clara de los posibles factores etiológicos.

- Parece importante el diagnóstico precoz de estos casos. Los autores del trabajo y otras publicaciones [2] refieren discretamente que los casos de menor evolución tienen mejor pronóstico, aunque este dato debe concretarse más en proximos estudios.
- La ayuda de la RM parece importante para confirmar de forma incruenta y rápida esta posibilidad diagnóstica. La mielografia, aunque muy útil, será posiblemente desbordada en los próximos años.

- El tratamiento endovascular es una buena alternativa a la cirugía y puede ser iniciado inmediatamente tras el diagnóstico arteriográfico. Laintroducción de líquidos adhesivos que trombosen parece más favorable que los émbolos, si bien serán caminos que deben decidirse en función de los resultados. La cirugía puede, así, ser un segunda opción, ante un fracaso de la terapia endovascular o participación de la arteria espinal anterior [2].

- Debe diferenciarse claramente la mejoría radiológica de la fístula y la mejoría clínica de los pacientes, ya que son éxitos no siempre simultáneos. La correcta valoración de los pacientes en el período previo y posterior es una misión del neurólogo clínico y es el indicador de la eficacia de nuestros tratamientos.

\section{BIBLIOGRAFÍA}

1. Merland JJ, Riche MC. Chiras J. Les fistules artérioveineuses intracanalaires, extra-médullaires à drainage veineux médullaire. J Neuroradiology 1980; 7: 271-320.

2. Huffmann BC, Gilsbach JM, Thron A. Spinal dural arteriovenous fistulas: A plea for neurosurgical treatment. Acta Neurochir (Wien) 1995; 135: 44-51. 